\title{
Characterization of a hybrid scanning system comprising acousto-optical deflectors and galvanometer scanners
}

\author{
Daniel Franz ${ }^{1}$ (D) Tom Häfner $^{2} \cdot$ Tim Kunz $^{2} \cdot$ Gian-Luca Roth $^{1} \cdot$ Stefan Rung ${ }^{1} \cdot$ Cemal Esen $^{3} \cdot$ Ralf Hellmann $^{1}$
}

Received: 29 November 2021 / Accepted: 11 February 2022 / Published online: 25 February 2022

(C) The Author(s) 2022

\begin{abstract}
We report on the characterization of a hybrid laser scanning system using acousto-optical deflectors in combination with galvanometer scanners for ultra-short pulse laser material processing. The hybrid scanning system is characterized by the roundness of static pulsed ablations of metal thin film on a transparent substrate within the acousto-optical scanning field at different galvanometer scanner deflection angles and laser focal positions. An ablation roundness of more than $90 \%$ is reached in a defocusing range of $200 \mu \mathrm{m}$ within a galvanometer scanfield of $900 \mathrm{~mm}^{2}$, corresponding to approximately $74 \%$ of the usable scan area of the $f$-theta lens. A high maximum positioning speed of $843 \mathrm{~m} / \mathrm{s}$ is pointed out within an acoustooptical scanfield of $0.4 \mathrm{~mm}^{2}$ by applying positioning frequencies of up to $1 \mathrm{MHz}$ across a distance of $843 \mu \mathrm{m}$. Consequently, the hybrid scanning system combines the advantages of optical and mirror-based scanners, enabling a highly dynamic and extremely precise laser beam positioning in a large processing area.
\end{abstract}

\section{Introduction}

Ultra-short pulse (USP) lasers emit pulses in the pico- and femtosecond range and have gained particular significance in high-precision micro-material processing due to negligible thermal load, often attributed to the so-called cold ablation $[1,2]$. Thus, USP lasers are typically used in electronics manufacturing [3-5], medical technology [6-10], aerospace applications [11-13], thin film technology [14] and material modification [15-20]. Although excellent processing qualities are possible, a broad adoption in industrial applications is constrained by low processing speeds and consequently limited productivity [21].

Assuming constant ablation efficiency, the productivity of the USP ablation process is determined by the applied average laser power [22]

Daniel Franz

Daniel.Franz@th-ab.de

1 Applied Laser and Photonics Group, University of Applied Sciences Aschaffenburg, Würzburger Straße 45, 63743 Aschaffenburg, Germany

2 Schmoll Maschinen GmbH, Odenwaldstraße 67, 63322 Rödermark, Germany

3 Applied Laser Technologies, Ruhr University Bochum, Universitätsstraße 150, 44801 Bochum, Germany
$P_{\text {avg }}=f \cdot E$,

which is in turn defined as the product of the laser pulse repetition rate $f$ and the laser pulse energy $E$. This implies two different ways for enhancing the productivity:

- Using a higher laser pulse repetition rate

- Processing with higher laser pulse energy.

Since a fluence of a few $\mathrm{J} / \mathrm{cm}^{2}$ is frequently sufficient for high-precision micromachining, static beam shaping optics, i.e. using diffractive optical elements (DOEs) [23-25] or dynamic beam shaping using Spatial Light Modulators (SLMs) [26, 27], are commonly used for splitting the laser beam into several partial sub-beams, enabling a parallel processing [22].

Alternatively, for increasing the productivity of USP laser ablation processes, a higher laser pulse repetition rate can be employed. However, the impact of pulse to pulse interactions on process efficiency [28, 29], thermal load [21, 30] and consequently precision $[31,32]$ as well as ablation behavior [33] have to be considered. Pulse to pulse interactions can be generally divided into optical interactions with laser-induced plasma or ablation particles and heat accumulation [21]. For counteracting these effects, a reduction of the spatial laser pulse overlap by an appropriate fast laser beam scanning system is necessary. The combination of typical available USP 
laser repetition rates of more than $10 \mathrm{MHz}[21,34]$ and the requirement of a local separation of the laser pulses poses challenges for commercially used 2D galvanometer scanners, as their dynamic range is limited by the inertia of the moving parts [35]. Therefore, alternative laser beam deflection technologies are being explored and tested [35-39].

Laser scanning technologies are divided into vector-based and pixel-based scanning. In pixel-based scanning, e.g. using polygon scanners, very high deflection speeds of more than $100 \mathrm{~m} / \mathrm{s}$ [40-43] are reachable by deflecting a laser beam along one axis using a continuously rotating polygon wheel [44]. As a result, pixelation is arising at a constant scanning speed and laser pulse repetition rate applying pulsed laser sources. Therefore, raster-based scanning technologies are suitable for large-area laser processing but not for high-precision laser beam positioning, e.g. required for laser drilling applications.

In contrast to this, using a vector-based scanning system, a high precision is accessible by moving the laser beam along the defined vector trajectory. A disadvantage of mirror-based scanning systems, such as piezoelectric, MEMS and galvanometer scanners, is a limited dynamic due to the inertia of the moving parts [35]. Therefore, optical deflectors, such as acousto-optical deflectors (AODs) or electro-optical deflectors (EODs), are of great interest for laser beam scanning as their deflection is based on the principle of diffraction, caused by a periodic refractive index modulation in an optically transparent medium, which in turn is the result of inducing acoustic waves or an electric field [35, 45], respectively.

Overall performances of EODs are comparable to AODs, except of a relatively high power absorption and an electrical power dissipation into the optical crystal, which can cause thermal lensing and consequently inaccuracies in laser beam positioning. In addition to that, maximum EOD aperture sizes of typically $2 \mathrm{~mm}$ [35] limit the focusability of the laser beam hence, AODs are preferred for processing with small laser focal diameters.

Two orthogonal arranged acousto-optical deflectors are required to form a 2D scanning unit, as a single AOD deflects a laser beam only in one plane. With a 2D AOD subsystem, highly dynamic laser beam deflection is feasible in a small deflection angle range of typically $\Theta=0.01-0.05 \mathrm{rad}$ [35]. To extend the deflection angle of the AOD subsystem by a factor of up to 100 [35], acousto-optical deflectors are combined with two galvanometer scanners, whereby a laser beam deflection in a scanfield of usually $10,000 \mathrm{~mm}^{2}$ [46] is reachable.

Bechtold et al. [37] applied a hybrid scanning system in combination with a $f$-theta lens for microstructuring of PMMA. An acousto-optical scanning speed of $26 \mathrm{~m} / \mathrm{s}$ $(806 \mathrm{rad} / \mathrm{s})$ is demonstrated, which is around 13 times faster as compared to the maximum deflection speed of the employed galvanometer scanners. Using a similar experimental setup, Eifel [36] achieved laser beam scanning speeds of up to $140 \mathrm{~m} / \mathrm{s}$ with an AOD subsystem and demonstrates the separation of $20 \mu \mathrm{m}$ diameter laser focal spots at laser pulse repetition rates up to $7 \mathrm{MHz}$.

The importance of high-speed processing using AODs in laser micropatterning of embossed metal rolls has been demonstrated by Bruening et al. [39, 47]. Positioning picosecond laser pulses with an AOD deflection speed of $17 \mathrm{~m} / \mathrm{s}$, a complete pulse to pulse separation was accomplished at a laser pulse repetition rate of $2 \mathrm{MHz}$ by rotating the embossed metal with a constant speed of $1.3 \mathrm{~m} / \mathrm{s}$. Laser pulses were separated down to a spatial pulse spacing of $8.5 \mu \mathrm{m}$ using a laser focal diameter of $9 \mu \mathrm{m}$. The maximum ablation rate of $3 \mathrm{~mm}^{3} / \mathrm{min}$ is an order of magnitude larger as compared to a typical 2D galvanometer scanner [48].

Besides full separation of ultra-short laser pulses, AODs allow simultaneously modulation of the laser beam intensity and are also used for compensation of vibrations and overshoots during fast mirror movements reducing processing time and enhancing machining quality $[49,50]$.

A proof of concept of hybrid scanning systems consisting of AODs and galvanometer scanners has already been presented [36, 51, 52]. However, superimposed scanning systems have not been characterized to determine their application potential for high-dynamic laser micromachining with USP lasers. Therefore, in this study, a scanning system consisting of two AODs in combination with a 2D galvanometer scanner is comprehensively characterized by evaluating static pulsed ablations of metal thin film on transparent substrate. The dynamic and precision of the AOD subsystem is determined at different positioning speeds within its scanfield. Furthermore, static pulsed ablations of AOD grids using different galvanometer deflections and focal planes are analyzed to characterize the hybrid scanning system in complete.

\section{Experimental}

\subsection{Hybrid setup}

The experimental setup for characterizing the superimposed laser beam deflection using two orthogonal AODs (Gooch \& Housego, AODF 4140 quartz $532 \mathrm{~nm}$ ) in combination with galvanometer scanners (Newson, RTA-AR800-3G) is illustrated in Fig. 1. Laser beam positioning by the AOD subsystem is based on the principle of diffraction. For filtering the +1 st diffraction order of the laser beam after passing of the AODs, two $4 f$ setups with lenses of the focal lengths of $f_{1}=200 \mathrm{~mm}, f_{2}=400 \mathrm{~mm}$ and $f_{3}=500 \mathrm{~mm}$ in combination with apertures are applied. Before entering an AOD, a half-wave plate is used to adapt 


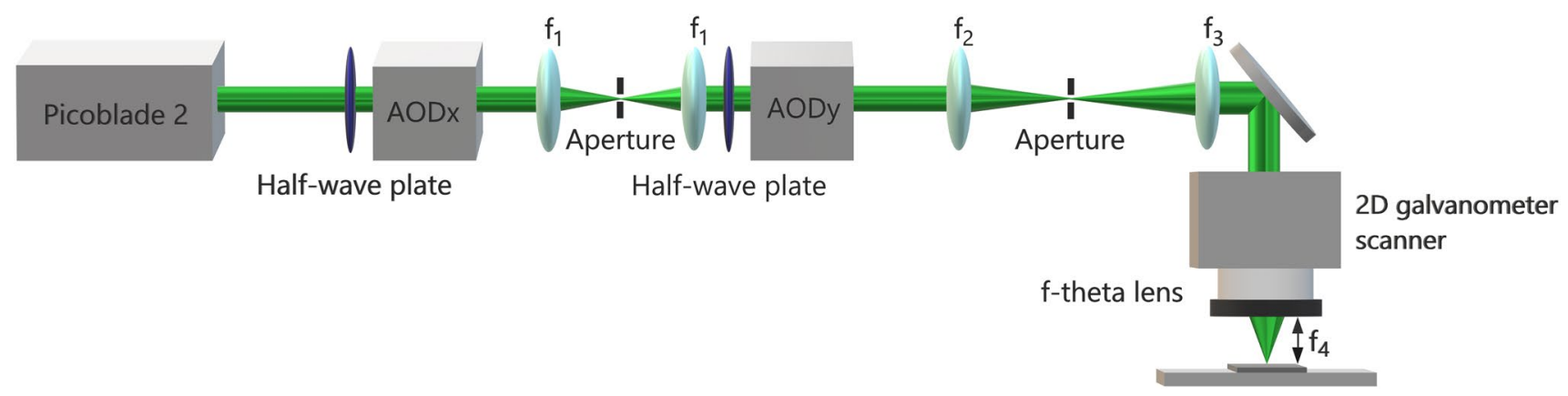

Fig. 1 Experimental setup for analyzing the superimposed laser beam deflection consisting of two acousto-optical deflectors and a 2D galvanometer scanner

the linear polarization state of the laser for minimizing the diffraction-induced loss of the system. The AOD subsystem, which includes the lenses of the $4 f$ setups and the telecentric $f$-theta lens (Sill Optics, S4LFT4010/292) with a focal length of $f_{4}=100 \mathrm{~mm}$, enable high-speed laser positioning in a range of about $0.4 \mathrm{~mm}^{2}$. For exploiting the complete scanning range of the $f$-theta lens up to $1225 \mathrm{~mm}^{2}$, a second beam deflection by a 2D galvanometer scanner is superimposed on the acousto-optical laser beam deflection. An USP laser (Lumentum, Picoblade 2) with a wavelength of $\lambda=532 \mathrm{~nm}$, a laser pulse duration of $\tau=10 \mathrm{ps}$ and a beam quality of $M^{2}=1.2$ was applied for experimental investigations using a laser pulse repetition rate of $f=2.2 \mathrm{MHz}$.

\subsection{Performance of the AOD subsystem}

One key specification of an acousto-optical deflection system is the diffraction efficiency (DE), generally defined as the relation of the laser power of the 0 th to the +1 st diffraction order. Therefore, for analyzing the DE profile of the presented AOD subsystem, five representative acoustooptical modulation frequencies and consequently deflection angles were applied for each AOD. To determine the 0th diffraction order, the laser power was measured with a power meter after passing through the AODs under continuous stimulation by the central modulation frequency of $140 \mathrm{MHz}$. Subsequently, after filtering by the $4 f$ setups, the laser power of the +1 st diffraction order was determined using modulation frequencies within the bandwidth of the AODs, cf. Fig. 1. Overall, an averaged DE of $(68.6 \pm 2) \%$ is reached for the AOD subsystem, whereby a deviation of up to $8.9 \%$ is identified applying modulation frequencies in a range of 110-170 MHz, cf. Fig. 2. The diffraction-induced deviations can be evaded by adapting the amplitudes for different modulation frequencies with the associated software. However, this was not performed in the experimental investigations.

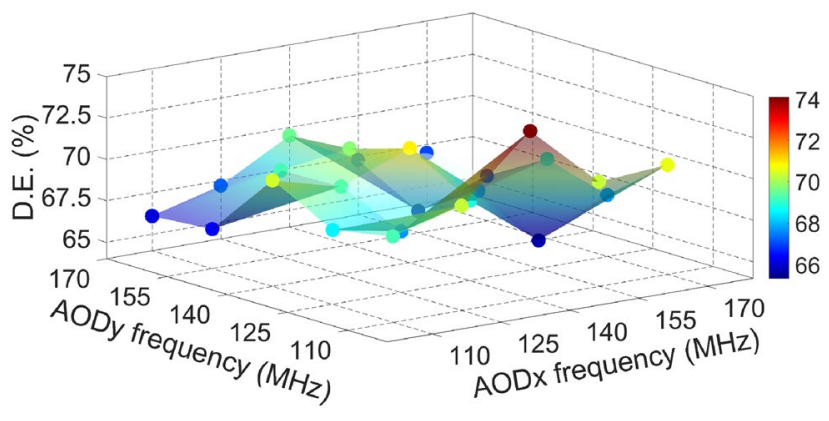

Fig. 2 DE profile of the AOD subsystem within its bandwidth at 25 representative deflection angles

\subsection{Methods}

In this study, static pulsed ablations of $110 \mathrm{~nm}$ metallic thin films on a transparent substrate are used for the characterization of the superimposed beam deflection. Thereby, the applied laser fluence on the sample level was adjusted close to the ablation threshold of the material to detect a modification induced by the hybrid scanning system, whereby an averaged ablation with a diameter of $13.4 \mu \mathrm{m}$ and a roundness of $95.3 \%$ is achieved at zero deflection of the AODs using galvanometer deflections in the applicable range of the $f$-theta lens. The geometry of the material removal is analyzed by transmission light microscopy (Leica DM6000 M) and can be used for the determination of the laser beam intensity distribution at defined laser focal positions [53]. Based on this approach, the characterization of the hybrid scanning is performed by three experimental procedures.

Both, dynamics and accuracy of the positioning of the AOD subsystem are studied by the generation of two diagonal ablations within an AOD scanfield at position 1 and 2 applying varying positioning frequencies between 0.2 and $1 \mathrm{MHz}$, cf. Fig. 3a. The galvanometer scanners are static at the central position during this process. For detection of deviations in laser beam positioning using the AOD subsystem, each ablation position is scanned once 


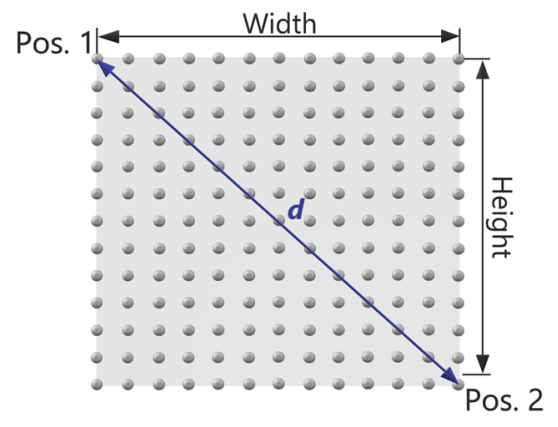

(a) AOD grid

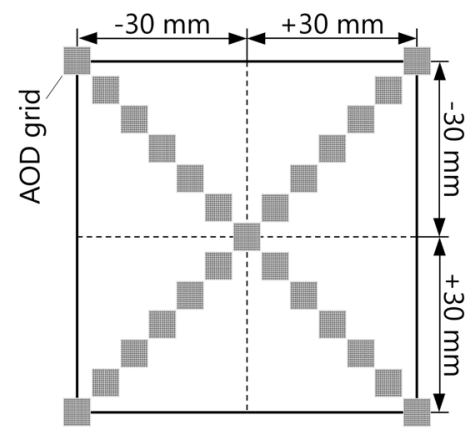

(b) Galvanometer deflections

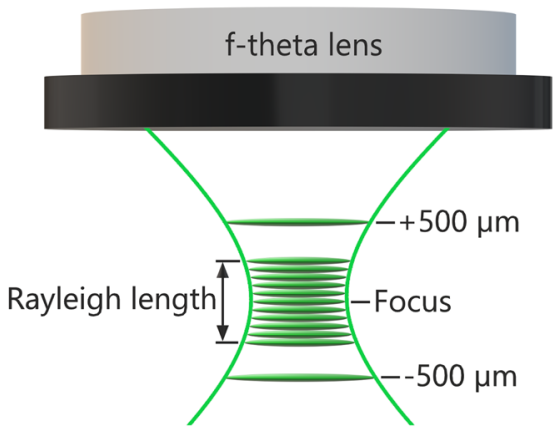

(c) Focal positions

Fig. 3 Experimental investigations for characterizing the superimposed laser beam deflection of a hybrid scanning system consisting of AODs and galvanometer scanners

within a single cycle of AOD scanning, whereby one pulse is emitted on the transparent substrate. As the generation of each single ablation spot requires 30 laser pulses, 30 cycles of AOD scanning are applied. Both, the diameters of the ablation spots and the distance $d$ between the ablation centers are evaluated. The smaller the fluctuation of both parameters, the higher the positioning accuracy of acousto-optical scanning system.

Key requirements for micro-material processing are a constant laser beam profile inside the processing area and an accurate positioning in the focal plane of the $f$-theta lens. For detecting a variation of the Gaussian intensity profile of the laser spot in the focal plane of the $f$-theta lens, AOD ablation grids with $13 \times 13$ equidistant arranged spots were fabricated at different galvanometer deflections in a range of $\pm 30 \mathrm{~mm}$, cf. Fig. 3b. Ablation grids are analyzed with respect to the roundness of individual ablation spots and to the width and height of an AOD scanfield, which are defined by the horizontal and vertical distance between the two outermost ablation centers of a grid, cf. Fig. 3a. The roundness of an ablation is defined by the ratio of minimum and maximum diameter.

Another prerequisite of a laser scanning system are precisely defined beam propagation properties along focusing, often specified by the so called Rayleigh length. The Rayleigh length is defined by

$z_{r}=\frac{\pi \cdot w_{f}^{2}}{\lambda}$

where $w_{f}$ is the radius of the beam waist $\left(1 / \mathrm{e}^{2}\right)$ and $\lambda$ is the wavelength of the laser. The Rayleigh length represents a characteristic distance over which the laser beam waist expanded by a factor of $\sqrt{2}$ leading to halving of the laser intensity $[54,55]$. In turn, the radius of the laser beam waist can be calculated by $w_{f}=\frac{\lambda \cdot f \cdot M^{2}}{w_{0} \cdot \pi}$,

where $\lambda$ is the wavelength of the laser, $f$ defines the focal length of the focusing lens, $w_{0}$ is the laser beam radius on the lens $\left(1 / \mathrm{e}^{2}\right)$ and $M^{2}$ represents the beam quality of the laser. In this study, a raw beam radius of $w_{0}=2.2 \mathrm{~mm}$ was measured by a CMOS camera (IDS uEye UI-1490SE) mounted prior to the 2D galvanometer scanner. Subsequently, a laser focal radius of $w_{f}=9.2 \mu \mathrm{m}$ was calculated by substituting the experimental used data in (3), which in turn results in a Rayleigh length of about $500 \mu \mathrm{m}$ from (2).

Metallic thin film ablations were generated in transparent substrate in steps of $50 \mu \mathrm{m}$ within the Rayleigh length and at two $z$-planes of $\pm 500 \mu \mathrm{m}$ with respect to the focal plane of the $f$-theta lens depending on five representative galvanometer deflections, cf. Fig. 3c. In turn, the roundness of the ablations within an AOD scanfield and its dimensions are analyzed.

\section{Results and discussion}

\subsection{Dynamics and precision of the AOD subsystem}

For determination of the maximum deflection dynamics and precision of the AOD subsystem, two diagonal metallic thin film ablations were alternately generated in transparent substrate within an AOD scanfield across a distance $d$ at galvanometer non-deflection using different positioning frequencies, cf. Fig. 3a.

The results of measured ablation distances $d$ as a function of the positioning frequencies in a range of $0.2-1 \mathrm{MHz}$ are depicted in Fig. 4a. The average distance between ablation position 1 and 2 is $843.4 \mu \mathrm{m}$ with a maximum deviation of $0.3 \mu \mathrm{m}$ and is independent of the applied positioning 


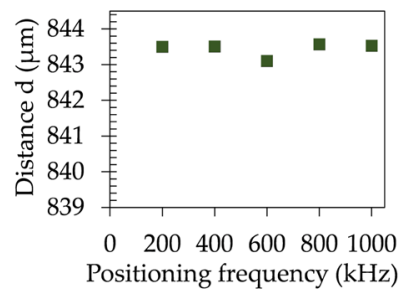

(a) Spot distance

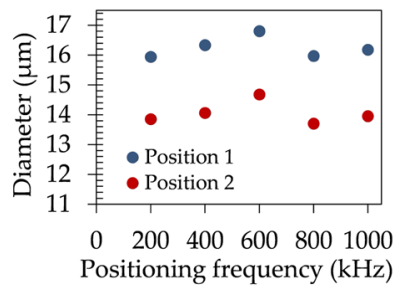

(b) Diameter
Fig. 4 Evaluation of the dynamics and the precision of a 2D acoustical scanning system combining two orthogonally arranged AODs

frequency. Furthermore, the diameter of the ablations were determined to detect positioning deviations by multiple repositioning. The ablation diameters in dependence of the positioning frequencies are illustrated in Fig. 4b. The diameter of ablations at positions 1 and 2 is determined with a mean value of $d_{1}=16.2 \mu \mathrm{m}$ and $d_{2}=14 \mu \mathrm{m}$, respectively. The standard deviation of the diameters is $0.3 \mu \mathrm{m}$ resulting from measurement inaccuracies and is not caused by positioning deviations of the AOD subsystem. The different ablation diameters at position 1 and 2 are created by a not calibrated DE profile of the AODs, cf. Fig. 2, whereby a smaller or larger amount of the Gaussian intensity distribution of the laser pulses leads to material ablation owing to different laser fluences. A constant DE within an AOD scanfield can be achieved by adjustment of the amplitudes for different modulation frequencies.

Altogether, the positioning frequency of the AOD subsystem is demonstrated up to $1 \mathrm{MHz}$ (time interval $=1 \mu \mathrm{s}$ ) across a distance of $843 \mu \mathrm{m}$, corresponding to a maximum positioning speed of $843 \mathrm{~m} / \mathrm{s}$. In the study, for the used beam diameter of $w_{d}=4.4 \mathrm{~mm}$, maximal positioning frequency of the AODs is reached as the sound waves requires at a sound velocity of $5740 \mathrm{~m} / \mathrm{s}$ about $1 \mu$ s for the generation of the refractive index modulation within the necessary active area of the acousto-optical medium of $5.7 \mathrm{~mm}$ (central laser beam propagation through the optical AOD aperture of $7 \mathrm{~mm}$ ).

\subsection{Characterization in the focal plane}

For characterization of the superimposed beam deflection consisting of an AOD subsystem and a 2D galvanometer scanner, the roundness of metallic thin film ablations within an AOD scanfield and their dimensions were determined at different galvanometer deflections in the focal plane of the $f$-theta lens. For the following roundness evaluations, typically industrial roundness specifications of micro-holes of $>90 \%$, e.g. of microvias [56], were used for comparison.

The obtained averaged roundness of ablations and their standard deviations within an AOD scanfield are illustrated in Fig. 5a using galvanometer deflections in a range of $\pm 30 \mathrm{~mm}$. Industrial requirements on ablation roundness

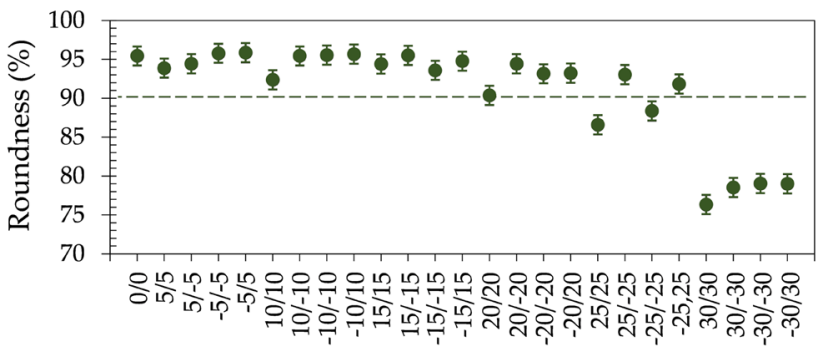

Galvanometer deflection $\mathrm{X}, \mathrm{Y}(\mathrm{mm})$

(a) Roundness of ablations within the AOD scanfields

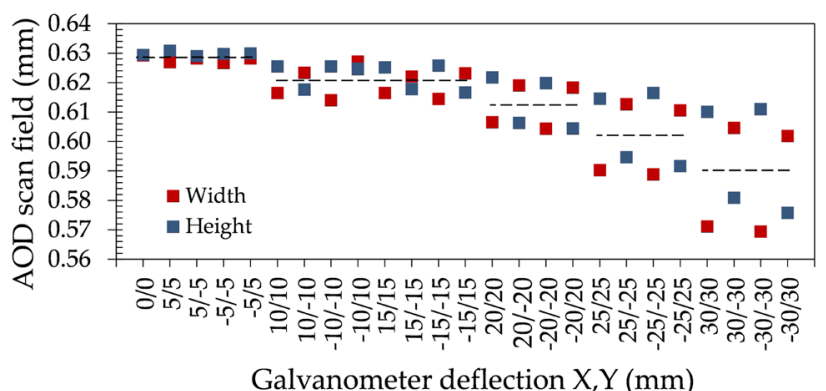

(b) Width and Height of the AOD scanfields

Fig. 5 Roundness of metallic thin film ablations within an full AOD scanfield and its dimensions in the focal plane of the $f$-theta lens depending on the galvanometer deflection

of $>90 \%$ within an AOD scanfield are feasible applying galvanometer deflections in a range of $\pm 15 \mathrm{~mm}$ with an average roundness of $(94.8 \pm 1.6) \%$. The averaged roundness decreases by $2 \%$ to $92.8 \%$ using galvanometer deflections of $\pm 20 \mathrm{~mm}$. The roundness of the ablations in the AOD grid is consistently lower in the upper right deflection range compared to the other galvanometer deflections, cf. Fig. 5a, hence roundness specifications cannot be completely fulfilled from galvanometer deflections of $\pm 20 \mathrm{~mm}$. This effect is mainly attributed to insufficient centric and parallel propagation of the laser radiation through the $f$-theta lens. For the remaining galvanometer deflections in a range of $\pm 20 \mathrm{~mm}$, industrial specifications are sufficiently fulfilled with an averaged roundness of $93.6 \%$, though the working range of the $f$-theta lens is merely $\pm 17.5 \mathrm{~mm}$.

For larger galvanometer deflections of $\pm 25-30 \mathrm{~mm}$, roundness drops to an average of $84.1 \%$, as telecentric correction is not given outside the operating zone of the $f$-theta lens. Hence, at larger galvanometer deflections from $\pm 25 \mathrm{~mm}$, an increasing distortion of the AOD scanfields is evident, which is noticeable in the roundness of the resulting metal thin film ablations, cf. Fig. 6f, g.

Additionally, the widths and heights of the AOD scanfields were determined depending on the galvanometer deflection in a range of $\pm 30 \mathrm{~mm}$ in the focal plane of the $f$-theta lens. At galvanometer deflections of $\pm 5 \mathrm{~mm}$, a constant AOD 

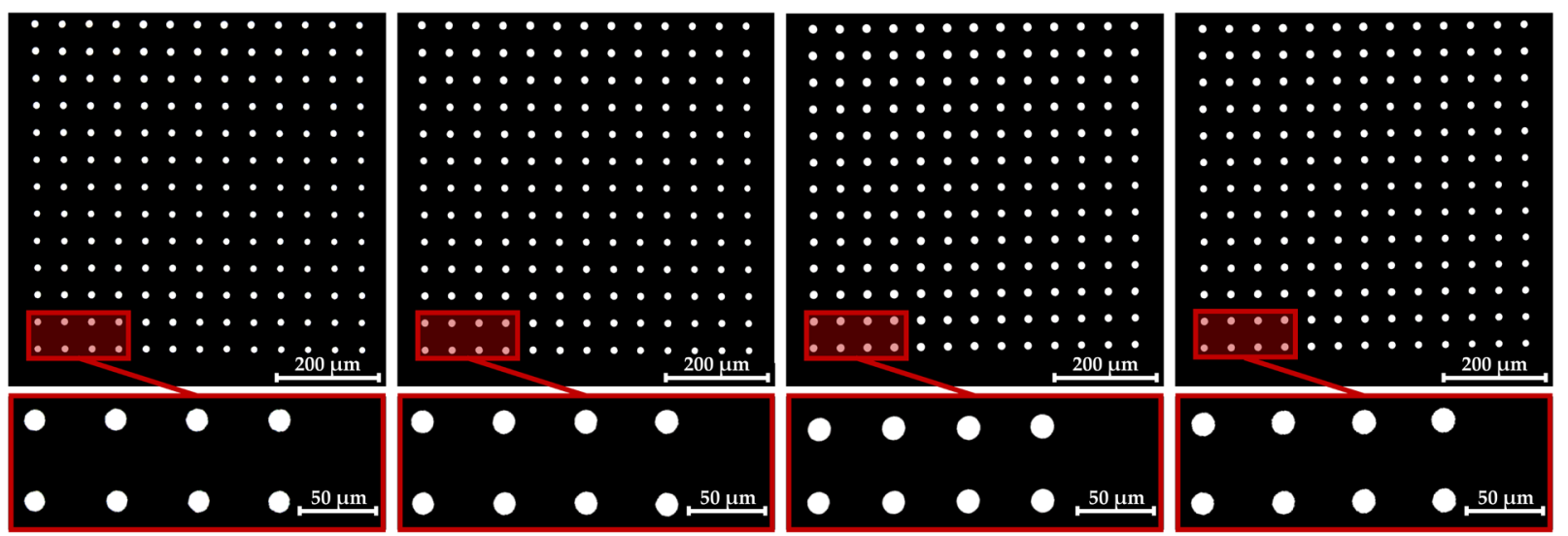

(a) $0 / 0 \mathrm{~mm}$

(b) $+5 /-5 \mathrm{~mm}$

(c) $+10 /-10 \mathrm{~mm}$

(d) $+15 /-15 \mathrm{~mm}$

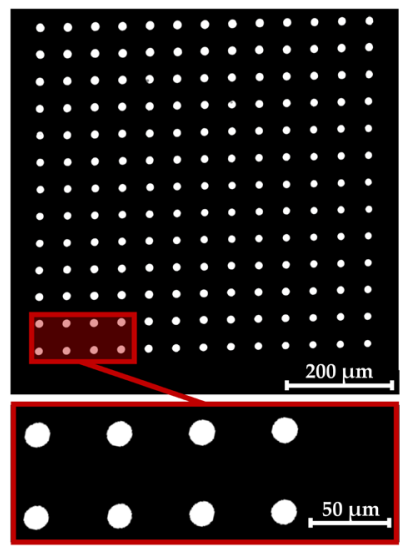

(e) $+20 /-20 \mathrm{~mm}$

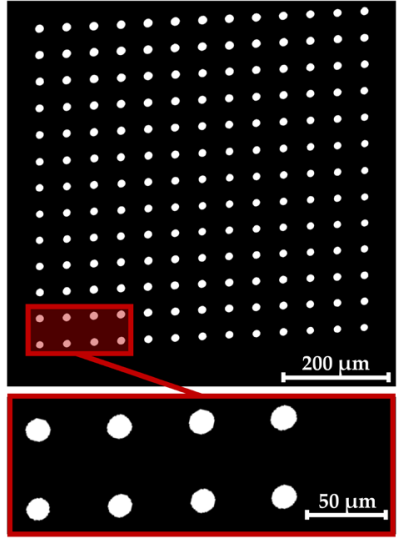

(f) $+25 /-25 \mathrm{~mm}$

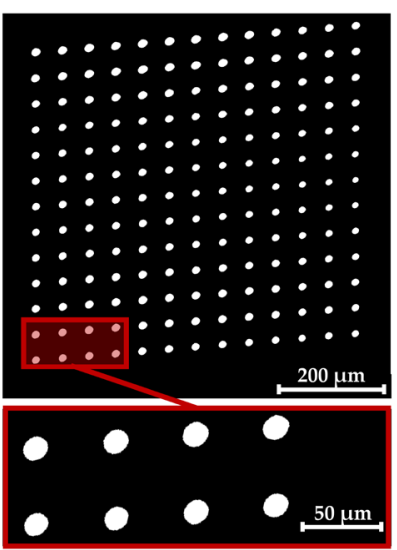

(g) $+30 /-30 \mathrm{~mm}$

Fig. 6 Processing results of metal thin film ablations on transparent substrate using transmitted light microscopy. Each picture a-g shows the ablation spots within a single AOD scanfield at selected galvanometer deflections

scanfield size of averaged $628.7 \mu \mathrm{m}$ is achieved. For larger galvanometer deflections, the AOD scanfield size decreases. The average width and height of the AOD scanfield declines using galvanometer deflections in the range of $\pm 10-15 \mathrm{~mm}$ to $(624 \pm 3.9) \mu \mathrm{m}$ and $(620.3 \pm 5.3) \mu \mathrm{m}$. Averaged AOD scanfield sizes of $612.6 \mu \mathrm{m}$ and $602.5 \mu \mathrm{m}$ with significantly larger standard deviations of $7.7 \mu \mathrm{m}$ and $11.1 \mu \mathrm{m}$ were obtained at galvanometer deflections in the range of 20 and $25 \mathrm{~mm}$, respectively. The sharply decreasing AOD scanfield dimensions are the result of the increasing distortion from galvanometer deflections of $\pm 25 \mathrm{~mm}$, evident in the processing results of metallic thin film ablations within an AOD scanfield depicted in Fig. 6.

In summary, industrial roundness specifications of $>90 \%$ are achieved within an complete AOD scanfield using galvanometer deflections of $\pm 15 \mathrm{~mm}$, which is exemplary illustrated by SEM images in Fig. 7 for a galvanometer deflection of $+15 \mathrm{~mm} /+15 \mathrm{~mm}$. Compared to the reference measurements without acousto-optical deflection, only minor averaged deviations of the ablation diameter of $0.8 \mu \mathrm{m}$ and roundness of $0.5 \%$ within an AOD scan field are demonstrated applying galvanometer deflections in the usable range of the $f$-theta lens. Therefore, for extreme galvanometer deflections, deviations in positioning are attributed to the $f$-theta lens. However, to fulfill high requirements in positioning accuracy in laser micromachining, AOD scanfield dimensions have to be scaled with the galvanometer deflection, which is feasible by adjusting the acoustic wave frequencies for defined positions.

\subsection{Characterization within the Rayleigh length}

Another fundamental specification for a laser beam scanning system in laser micromachining are well-defined laser beam propagation properties along focusing. Therefore, metallic thin film ablations were analyzed with respect to the roundness and the AOD scanfield dimensions using different laser focal positions, both within and beyond of the Rayleigh length, at five representative galvanometer deflections in a range of $\pm 25 \mathrm{~mm}$.

The averaged roundness of the metallic thin film ablations within an AOD scanfield and its dimensions depending on 

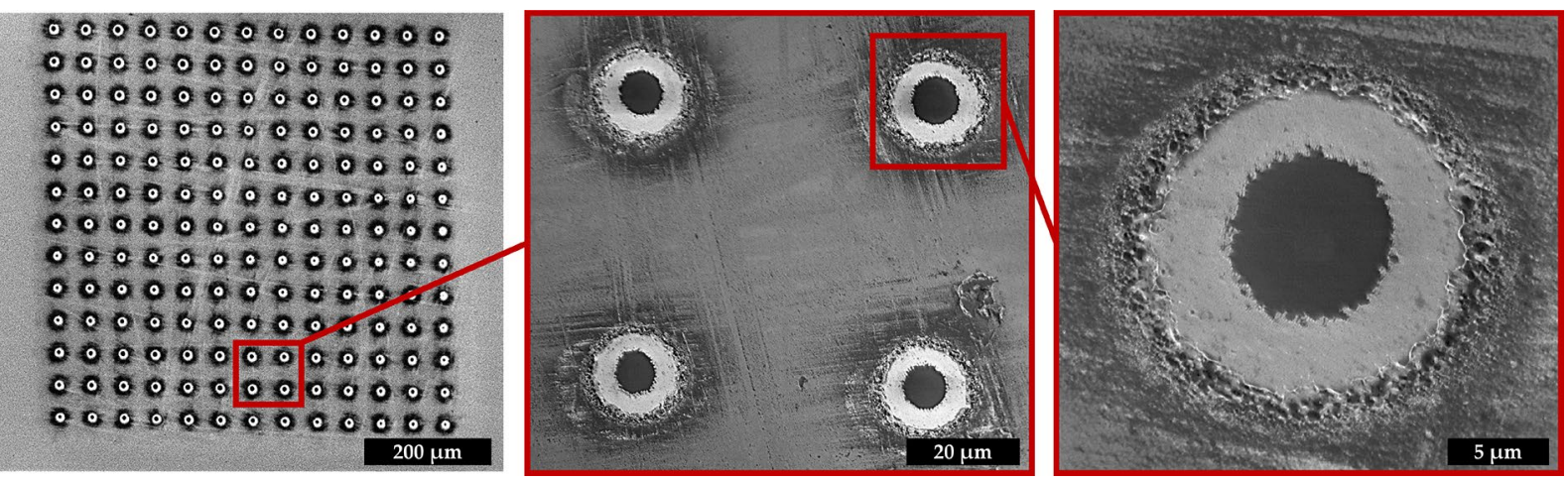

Fig. 7 REM images of metallic thin film ablations on a transparent substrate within an AOD scanfield applying a galvanometer deflection of $+15 \mathrm{~mm} /+15 \mathrm{~mm}$ in top view

the galvanometer deflection and focal plane is depicted in Fig. 8, whereby AOD scanfield dimensions are defined as the average of its widths and heights. An averaged ablation roundness of $(94.2 \pm 1.2) \%$ is reached using galvanometer non-deflection in various focal planes ranging between $+200 \mu \mathrm{m}$ to $-100 \mu \mathrm{m}$ relative to the focal plane of the $f$-theta lens, cf. Fig. 8a. Maximum ablation roundness with an average of $96.7 \%$ is reached in a $50 \mu \mathrm{m}$ plane below the focal position. The AOD scanfield dimensions increase continuously within a $z$-level of $-100 \mu \mathrm{m}$ to $+200 \mu \mathrm{m}$ from $628.8 \mu \mathrm{m}$ to $632.3 \mu \mathrm{m}$.
The averaged ablation roundness and the AOD scanfield dimensions as a function of different $z$-levels both inside and outside the Rayleigh length at galvanometer deflections of $+15 /-15 \mathrm{~mm}$ and $-15 \mathrm{~mm} /+15 \mathrm{~mm}$ are depicted in Fig. $8 \mathrm{~b}$, c. The usable $z$-range applying the hybrid scanning system with a spot roundness of $>90 \%$ is reduced by up to $100 \mu \mathrm{m}$ to $-50 \mu \mathrm{m}$ to $+150 \mu \mathrm{m}$ regarding to the focal plane. Within the acousto-optical scanfield, averaged ablation roundness of $(94.7 \pm 1.3) \%$ is achieved. Compared to galvanometer nondeflection, AOD scanfield dimensions decrease by an average of $6.9 \mu \mathrm{m}$ to $624.3 \mu \mathrm{m}$. However, maximum deviation is

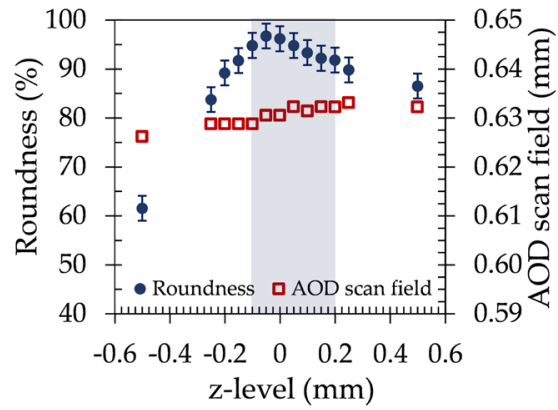

(a) $0 / 0 \mathrm{~mm}$

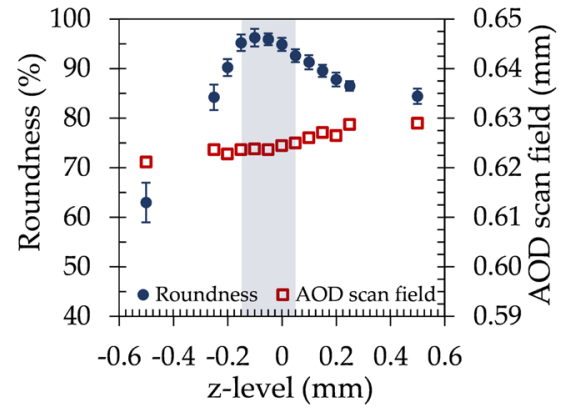

(b) $+15 /-15 \mathrm{~mm}$

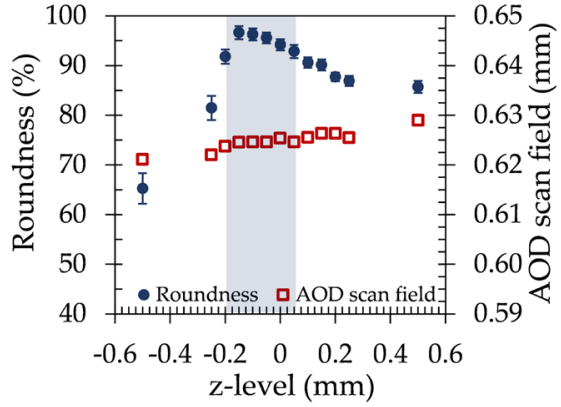

(c) $-15 /+15 \mathrm{~mm}$

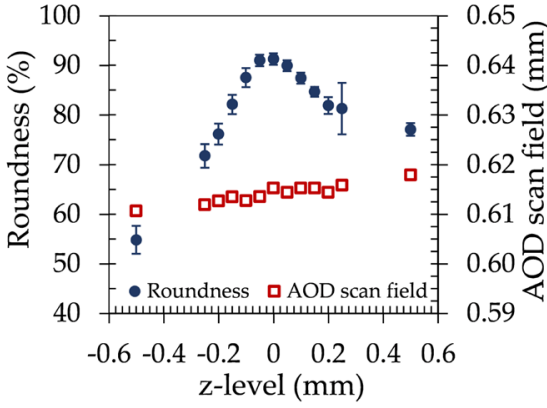

(d) $+25 /-25 \mathrm{~mm}$

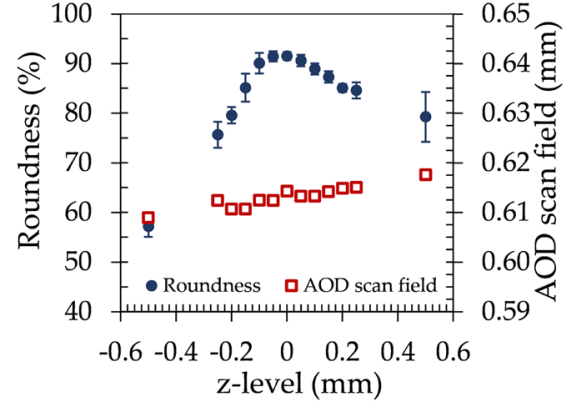

(e) $-25 /+25 \mathrm{~mm}$

Fig. 8 Roundness of metallic thin film ablations within an AOD scanfield and its dimensions depending on five representative galvanometer deflections and laser focal positions 
significantly reduced from $1.8 \mu \mathrm{m}$ to $0.9 \mu \mathrm{m}$. The roundness specifications of $>90 \%$ cannot be fulfilled at galvanometer deflections of $+25 \mathrm{~mm} /-25 \mathrm{~mm}$ and $-25 \mathrm{~mm} /+25 \mathrm{~mm}$, confirming the results of the previously outlined roundness analysis in the focal plane of the $f$-theta lens using different galvanometer deflections. Here, AOD scanfield dimensions are reduced by $2.8 \%$ to averaged $613.6 \mu \mathrm{m}$ compared to the galvanometer non-deflection.

All in all, roundness of ablations $>90 \%$ within an AOD scanfield is demonstrated within a $z$-range of $200 \mu \mathrm{m}$ at galvanometer deflections in a range of $\pm 15 \mathrm{~mm}$, sufficient for laser micromachining applications. However, for reaching the high requirements in laser beam positioning with the hybrid scanning system, AOD scanfield deviations of up to $6.9 \mu \mathrm{m}$ have to be considered depending on different galvanometer deflection. In addition, processing in the focal plane of the $f$-theta lens is mandatory due to AOD scanfield deviations of up to $3.5 \mu \mathrm{m}$ using galvanometer deflections in a range of $900 \mathrm{~mm}^{2}$.

\section{Conclusion}

In this study, superimposed laser beam deflection applying two acousto-optical deflectors in combination with a 2D galvanometer scanner was characterized based on the roundness of static pulsed ablations of metal thin film on transparent substrate. Within a galvanometer deflection range of $900 \mathrm{~mm}^{2}$, ablation roundness of more than $90 \%$ is demonstrated within an AOD scanfield and a $z$-range of $200 \mu \mathrm{m}$, corresponding to about $36 \%$ of the Rayleigh length and $74 \%$ of the usable deflection area. To fulfill the requirements on positioning accuracy of the hybrid scanning system, a scaling of the AOD scanfield dimensions depending on the galvanometer deflection as well as the focal position is necessary, as maximum deviations of up to $6.9 \mu \mathrm{m}$ are observed. Maximum positioning speeds of $843 \mathrm{~m} / \mathrm{s}$ are demonstrated within the scanfield of the AOD subsystem using positioning frequencies up to $1 \mathrm{MHz}$, which highlights the great potential of the hybrid scanning system for highly dynamic micromaterial processing using ultra-short pulse laser. However, an overall diffraction-induced loss of the AOD subsystem of about $31.4 \%$ is pointed out using different modulation frequencies.

Funding Open Access funding enabled and organized by Projekt DEAL.

Open Access This article is licensed under a Creative Commons Attribution 4.0 International License, which permits use, sharing, adaptation, distribution and reproduction in any medium or format, as long as you give appropriate credit to the original author(s) and the source, provide a link to the Creative Commons licence, and indicate if changes were made. The images or other third party material in this article are included in the article's Creative Commons licence, unless indicated otherwise in a credit line to the material. If material is not included in the article's Creative Commons licence and your intended use is not permitted by statutory regulation or exceeds the permitted use, you will need to obtain permission directly from the copyright holder. To view a copy of this licence, visit http://creativecommons.org/licenses/by/4.0/.

\section{References}

1. S.-S. Wellershoff, J. Hohlfeld, J. Güdde, E. Matthias, The role of electron-phonon coupling in femtosecond laser damage of metals. Appl. Phys. A 69(1), 99-107 (1999)

2. J. Hohlfeld, S.-S. Wellershoff, J. Güdde, U. Conrad, E. Matthias, V. Jähnke, Electron and lattice dynamics following optical excitation of metals. Chem. Phys. 251(1-3), 237-258 (2000)

3. S. Luzius, M. Sailer, C. Siebert, S. Russ, PCB drilling with high power picosecond lasers. Phys. Procedia 41, 732-726 (2013)

4. D. Lee, Picosecond IR pulsed laser drilling of copper-coated glass/ epoxy composite. IEEE Trans. Compon. Packag. Manuf. Technol. 7(12), 2066-2072 (2017)

5. R.V. Chkalov, D.A. Kochuev, K.S. Khorkov, A.A. Voznesenskaya, S.M. Arakelian, Precision formation of PCB topologies by femtosecond laser radiation. J. Phys. Conf. Ser. 1164(1) (2019)

6. A. Trautmann, G.L. Roth, B. Nujiqi, T. Walther, R. Hellmann, Towards a versatile point-of-care system combining femtosecond laser generated microfluidic channels and direct laser written microneedle arrays. Microsyst. Nanoeng. 5(1), 1-9 (2018)

7. A.H. Lutey, L. Gemini, L. Romoli, G. Lazzini, F. Fuso, M. Faucon, R. Kling, Towards laser-textured antibacterial surfaces. Sci. Rep. 8(1), 1-10 (2018)

8. C.H. Hung, F.Y. Chang, Curve micromachining on the edges of nitinol biliary stent by ultrashort pulses laser. Opt. Laser Technol. 90, 1-6 (2017)

9. F. Hendricks, R. Patel, V.V. Matylitsky, Micromachining of bioabsorbable stents with ultra-short pulse lasers, in Frontiers in Ultrafast Optics: Biomedical, Scientific, and Industrial Applications XV, vol. 9355 (2015)

10. A.Y. Sajjadi, K. Mitra, M. Grace, Ablation of subsurface tumors using an ultra-short pulse laser. Opt. Lasers Eng. 49(3), 451-456 (2011)

11. S. Milles, V. Vercillo, S. Alamri, A.I. Aguilar-Morales, T. Kunze, E. Bonaccurso, A.F. Lasagni, Icephobic performance of multiscale laser-textured aluminum surfaces for aeronautic applications. Nanomaterials 11(1), 135 (2021)

12. Z. Liu, F. Zhang, Y. Chen, H. Zhang, Y. Han, J. Liu, L. Huang, $X$. Liu, Electrochemical fabrication of superhydrophobic passive films on aeronautic steel surface. Colloids Surf. A Physicochem. Eng. Asp. 572, 317-325 (2019)

13. Y.H. Ng, S.W. Tay, L. Hong, Formation of icephobic surface with micron-scaled hydrophobic heterogeneity on polyurethane aerospace coating. ACS Appl. Mater. Interfaces 10(43), 37517-37528 (2018)

14. A. Risch, R. Hellmann, Laser scribing of gallium doped zinc oxide thin films using picosecond laser. Appl. Surf. Sci. 258(5), 1849-1853 (2011)

15. G.L. Roth, S. Rung, C. Esen, R. Hellmann, Microchannels inside bulk PMMA generated by femtosecond laser using adaptive beam shaping. Opt. Express 28(4), 5801-5811 (2020)

16. G.L. Roth, S. Hessler, S. Kefer, M. Girschikofsky, C. Esen, R. Hellmann, Femtosecond laser inscription of waveguides and Bragg gratings in transparent cyclic olefin copolymers. Opt. Express 28(12), 18077-18084 (2020)

17. K. Elango, J.S. Hoppius, L.M. Kukreja, A. Ostendorf, E.L. Gurevich, Studies on ultra-short pulsed laser shock peening of stainlesssteel in different confinement media. Surf. Coat. Technol. 397, 125988 (2020) 
18. H. Wang, Y. Kalchev, H. Wang, K. Yan, E.L. Gurevich, A. Ostendorf, Surface modification of NiTi alloy by ultrashort pulsed laser shock peening. Surf. Coat. Technol. 394, 125899 (2020)

19. K. Bergner, M. Müller, R. Klas, J. Limpert, S. Nolte, A. Tünnermann, Scaling ultrashort laser pulse induced glass modifications for cleaving applications. Appl. Opt. 57(21), 5941-5947 (2018)

20. R. Stoian, Volume photoinscription of glasses: three-dimensional micro-and nanostructuring with ultrashort laser pulses. Appl. Phys. A 126, 1-30 (2020)

21. J. Finger, M. Reininghaus, Effect of pulse to pulse interactions on ultrashort pulse laser drilling of steel with repetition rates up to $10 \mathrm{MHz}$. Opt. Express 22(15), 18790-18799 (2014)

22. J.T. Finger, Ph.D. Thesis, RWTH Aachen University, Aachen (2017)

23. J.I. Kato, N. Takeyasu, Y. Adachi, H.B. Sun, S. Kawata, Multiple-spot parallel processing for laser micronanofabrication. Appl. Phys. Lett. 86(4) (2005)

24. O. Haupt, V. Schütz, U. Stute, Multi-spot laser processing of crystalline solar cells, in Laser-Based Micro-and Nanopackaging and Assembly V, vol. 7921 (2011)

25. L. Büsing, Ph.D. Thesis, RWTH Aachen University, Aachen (2016)

26. Z. Kuang, D. Liu, W. Perrie, S. Edwardson, M. Sharp, E. Fearon, G. Dearden, K. Watkins, Fast parallel diffractive multi-beam femtosecond laser surface micro-structuring. Appl. Surf. Sci. 255(13-14), 6582-6588 (2009)

27. Z. Kuang, W. Perrie, D. Liu, S. Edwardson, J. Cheng, G. Dearden, K. Watkins, Diffractive multi-beam surface micro-processing using 10 ps laser pulses. Appl. Surf. Sci. 255(22), 9040-9044 (2009)

28. A. Ancona, S. Döring, C. Jauregui, F. Röser, J. Limpert, S. Nolte, A. Tünnermann, Femtosecond and picosecond laser drilling of metals at high repetition rates and average powers. Appl. Phys. A 234(21), 3304-3306 (2009)

29. A. Ancona, F. Röser, K. Rademaker, J. Limpert, S. Nolte, A. Tünnermann, High speed laser drilling of metals using a high repetition rate, high average power ultrafast fiber CPA system. Opt. Express 16(12), 8958-8968 (2008)

30. B. Bomschlegel, J. Koller, J. Finger, In-situ analysis of heat accumulation during ultrashort pulsed laser ablation. J. Laser Micro Nanoeng. 15(1), 1-7 (2020)

31. D. Brinkmeier, D. Holder, A. Loescher, C. Röcker, D.J. Förster, V. Onuseit, R. Weber, M.A. Ahmed, T. Graf, Process limits for percussion drilling of stainless steel with ultrashort laser pulses at high average powers. Appl. Phys. A 128(1), 1-9 (2022)

32. R. Weber, T. Graf, P. Berger, V. Onuseit, M. Wiedenmann, C. Freitag, A. Feuer, Heat accumulation during pulsed laser materials processing. Opt. Express 22(9), 11312-11324 (2014)

33. J. Schille, R. Ebert, U. Loeschner, P. Scully, N. Goddard, H. Exner, High repetition rate femtosecond laser processing of metals, in Frontiers in Ultrafast Optics: Biomedical, Scientific, and Industrial Applications X, vol. 7589 (2010)

34. J. Schille, L. Schneider, S. Mauersberger, S. Szokup, S. Höhn, J. Pötschke, F. Reiß, E. Leidich, U. Löschner, High-rate laser surface texturing for advanced tribological functionality. Lubricants 8(3), 33 (2020)

35. G.R.B.E. Römer, P. Bechtold, Electro-optic and acousto-optic laser beam scanners. Phys. Procedia 56, 29-39 (2014)

36. S. Eifel, Ph.D. Thesis, RWTH Aachen University, Aachen (2015)

37. P. Bechtold, R. Hohenstein, M. Schmidt, Beam shaping and highspeed, cylinder-lens-free beam guiding using acousto-optical deflectors without additional compensation optics. Opt. Express 21(12), 14627-14635 (2013)

38. S. Toyoda, Y. Sasaki, J. Kobayashi, Improvement of $200 \mathrm{kHz}$ optical beam scanner performance with multiple internal reflection. J. Eng. 2014(3), 81-82 (2014)
39. S. Bruening, G. Hennig, S. Eifel, A. Gillner, Ultrafast scan techniques for $3 \mathrm{D}-\mu \mathrm{m}$ structuring of metal surfaces with high repetitive ps-laser pulses. Phys. Procedia 12, 105-115 (2011)

40. J. Lopez, K. Mishchik, G. Mincuzzi, E. Audouard, E. Mottay, R. Kling, Efficient metal processing using high average power ultrafast laser. J. Laser Micro Nanoeng. 12(3), 296-303 (2017)

41. S. Bruening, K. Du, A. Gillner, Ultra-fast micro machining of cylindrical parts with multiple ultra-short pulsed laser sources. Phys. Procedia 83, 167-181 (2016)

42. U. Loeschner, J. Schille, A. Streek, T. Knebel, L. Hartwig, R. Hillmann, C. Endisch, High-rate laser microprocessing using a polygon scanner system. J. Laser Appl. 27(S2) (2015)

43. B. Jaeggi, B. Neuenschwander, M. Zimmermann, L. Penning, R. DeLoor, K. Weingarten, A. Oehler, High-throughput and high-precision laser micromachining with ps-pulses in synchronized mode with a fast polygon line scanner, in LAMOM XIX, vol. 8967 (2015)

44. O. Nottrodt, Ph.D. Thesis, RWTH Aachen University, Aachen (2017)

45. P.A. Kirkby, K.M.N.S. Nadella, R.A. Silver, A compact acoustooptic lens for 2D and 3D femtosecond based 2-photon microscopy. Opt. Express 18(13), 13720-13744 (2010)

46. M.A.O. Delgado, A.F. Lasagni, Reducing field distortion for galvanometer scanning system using a vision system. Opt. Lasers Eng. 86, 106-114 (2016)

47. K. Du, S. Bruening, A. Gillner, High-power picosecond laser with $40 \mathrm{~W}$ average power for large scale applications, in Laser-Based Micro- and Nanopackaging and Assembly VI, vol. 8244 (2012)

48. B. Jaeggi, B. Neuenschwander, M. Schmid, M. Muralt, J. Zuercher, U. Hunziker, Influence of the pulse duration in the psregime on the ablation efficiency of metals. Phys. Procedia 12 , 164-171 (2011)

49. P.A. Taschner, J. Düsing, J. Koch, P. Jäschke, S. Kaierle, L. Overmeyer, Divide-and-conquer laser beam deflection system: fast, wide-ranging, and flexible, in Laser-Based Micro-and Nanoprocessing XIV, vol. 11268 (2020)

50. G. Raciukaitis, Ultra-short pulse lasers for microfabrication: a review. IEEE J. Sel. Top. Quantum Electron. 27(6) (2021)

51. H. Matsumoto, M. Unrath, H. Zhang, B. Hainsey, Laser direct ablation for patterning printed wiring boards using ultrafast lasers and high speed beam delivery architectures. J. Laser Micro Nanoeng. 8(3), 315-320 (2013)

52. D. Franz, G.L. Roth, S. Rung, C. Esen, R. Hellmann, Superimposed beam deflection using acousto-optical deflectors in combination with a galvanometer scanner, in Lasers in ManufacturingLiM 2021 (2021)

53. S. Rung, J. Barth, R. Hellmann, Characterization of laser beam shaping optics based on their ablation geometry of thin films. Micromachines 5(4), 943-953 (2014)

54. K. Poder, J.M. Cole, J.C. Wood, S. Alatabi, P.S. Foster, C. Kamperidis, O. Kononenko, C.A. Palmer, D. Rusby, A. Sahai, G. Sarri, D.R. Symes, J.R. Warwick, S.P.D. Mangles, Z. Najmudin, Measurements of self-guiding of ultrashort laser pulses over long distances. Plasma Phys. Control Fusion 60(1) (2017)

55. A. Ajami, W. Husinsky, R. Liska, N. Pucher, Two-photon absorption cross section measurements of various two-photon initiators for ultrashort laser radiation applying the Z-scan technique. JOSA B 27(11), 2290-2297 (2010)

56. K.R. Kim, J.H. Cho, N.Y. Lee, H.J. Kim, S.H. Cho, H.J. Park, B. Choi, High-precision and ultrafast UV laser system for next-generation flexible PCB drilling. J. Manuf. Syst. 38, 107-113 (2016)

Publisher's Note Springer Nature remains neutral with regard to jurisdictional claims in published maps and institutional affiliations. 